\title{
Evidence-based Reconstruction of Kampo Medicine: Part-III-How Should Kampo be Evaluated?
}

\author{
Katsutoshi Terasawa
}

Toyama Medical and Pharmaceutical University, Japan

\section{Introduction}

In the previous two lectures, I tried to explain in detail that the paradigm of Kampo is basically different from that of modern Western medicine, focusing especially on the concept of Sho. Kampo has been developed to cope with Sho, not with the disease entities understood in terms of modern Western medicine. Thus, there is little point asking, for example, 'Are there any Kampo remedies effective for lung cancer (or type $\mathrm{C}$ hepatitis, or essential hypertension)?'

This question, asked wrongly, however, highlights two important issues, one intriguing and long term, and the other important and short term. The former is that the effects of Kampo herbs have so far not been tested properly against any of the specific disease entities defined in the framework of modern Western medicine. The latter is that we urgently need some methods to accommodate evaluation of the clinical effects of Kampo remedies in the framework of evidencebased medicine of the West.

\section{Kampo as a Frontier of Research on Complex Agents (drugs): a Long-term View}

It may sound paradoxical that the effects of various Kampo regimens so far have not been tested properly against any specific disease entities, given that these remedies have been in use for thousands of years for patients with a wide variety of disorders in East Asia. However, if we remember that the modern Western framework of diseases is only a couple of hundreds years old and people (patients and doctors alike) who used Kampo herbs had never been acquainted with any concepts such as lung cancer, type $\mathrm{C}$ hepatitis or angina pectoris,

For reprints and all correspondence: Professor Katsutoshi Terasawa,

Department of Japanese-Oriental (Kampo) Medicine, Faculty of

Medicine, Toyama Medical and Pharmaceutical University, Japan.

E-mail: terasawa@ms.toyama.ac.jp the issue raised here should become understandable. It has sometimes been claimed that the fact that Kampo medicine is effective is beyond doubt as it has endured thousands of years of 'clinical testing' by East Asian people. However, this is only so for Sho, or symptom conditions understood in the framework of Kampo. In other words, Kampo has been proven effective for Sho conditions, but not for any others. The effects of Kampo medicine on disease entities understood in the framework of modern Western medicine should be the subject of novel research. For example, in the first issue of eCAM, Kobayashi et al. (1) described in detail their clinical experience on the application of Kampo remedies for atopic dermatitis. As the very concept of atopic dermatitis is only half a century old, research on the effects of any Kampo agents on atopic dermatitis is basically brand new or, in other words, purely experimental. In that review, the authors reported their struggle to bring atopic dermatitis under control by various Kampo formulae. According to the authors, very few patients with atopic dermatitis refractory to modern Western remedies can be cured by one single formula of Kampo, but usually several formulae should be tried out against changing Sho, until the patients' symptoms are brought under control. Therefore, they say, it would be better to call their Kampo therapy a Kampo 'session', not a simple 'administration' of a single (or multiple) synthetic drug(s). This is a paradigmatic case to show that there are very few one-to-one correspondences between disease entities in modern Western terms and Kampo remedies. Kampo is not effective for atopic dermatitis in the way, for instance, that streptomycin is effective for tuberculosis. This situation has two intriguing implications. First, trying to find applications of Kampo medicine for 'modern Western' diseases itself is a venture into a new field. For example, it is a completely new quest to look for some Kampo remedies which are effective for, say, AIDS, type $\mathrm{C}$ hepatitis or prostate cancer. Secondly, in looking for applications of Kampo medicine to modern Western disease entities, it is not mandatory for a researcher to stay within the

The online version of this article has been published under an open access model. Users are entitled to use, reproduce, disseminate, or display the open access version of this article provided that: the original authorship is properly and fully attributed; the Journal and Oxford University Press are attributed as the original place of publication with the correct citation details given; if an article is subsequently reproduced or disseminated not in its entirety but only in part or as a derivative work this must be clearly indicated. 
framework of the traditional Kampo formulation. This may sound paradoxical, since of course some of the traditional formulae could be found to be effective for some disease entities, as indeed they have already been. However, there are other intriguing possibilities such as: (i) one particular chemical component in a Kampo herb may ultimately be found to be effective; (ii) one special composition of several chemical components in one kind of Kampo herb may be found to be effective; or (iii) a new combination of several traditional Kampo herbs may be found to be effective for some disease entities defined in the modern Western framework. In methodological terms, there are several procedures to pursue these possibilities. First, the effects of Kampo remedies can be tried out for as many disease entities as possible in pre-clinical settings, especially for diseases for which there is no effective synthetic chemical drug. Secondly, in doing that, researchers do not have to take the traditional formulae as invincible, as they have not been nurtured as agents for, for example, AIDS. A very 'reductionist' approach can even be taken to look for 'active component(s)' in the complex herbal formulae. Such approaches have already been being tried in various laboratories in Japan.

Kampo agents have not only not been tested properly for their effects against any modern disease entities, but they had also not been evaluated for their effects on any physiological, biochemical or immunological functions up until the middle of the last century. Thus, research such as the effects of Juzentaihoto on cytokine production published in the first issue of $e C A M$ (2) is among the first to be tried in the thousands of years of history of Traditional Chinese Medicine and Kampo. Basic chemical analyses of almost all the Kampo herbs had been nearly completed by 1980 in many laboratories especially in Japan, and research has flourished in looking at the effects of Kampo herbs on a variety of physiological, pharmacological, immunological and other parameters at the laboratory level. However, research into actions of many of Kampo component herbs and also various Kampo formulae is far from complete. As has been stressed in this lecture series $(3,4)$ and also in several other articles in $e C A M(5)$, the "composite' effects of Kampo herbs are the most important and interesting field of research for the future.

From one perspective, we can even see that the thousands of years of experience in East Asia have generated a vast volume of 'clinical suggestions' or 'epidemiological raw data' that some herbs or combination of herbs are effective on some diseases or some physiological processes. To determine what herbal component(s)/herbs/combination herbs are effective for what kind of diseases or what pathophysiological processes is essentially a new field of basic research. Given the complexity of Kampo herbs and their composite preparations, this is a long-term project, not much different from conventional clinicopharmaceutical research to determine effective antibacterial products from fungi and other organisms. The major difference between Kampo research and such a conventional approach is its attention to composite effects. Methodologies being developed for that aspect of Kampo will help conventional drug research to become more attentive to the possibility of such effects.

As a final note for this section, I would like to point out one more interesting aspect for long-term research on Kampo. Kampo agents, being herbal preparations, cannot only be regarded as 'drugs', but can also be seen as special 'foods', as exemplified by the famous Kampo slogan, 'Foods and drugs, from the same origin'. If so, 'effects' of Kampo agents can not only be evaluated from the viewpoint of drugs to 'cure' specific diseases, as for paradigmatic modern Western synthetic drugs. They can also be evaluated for their efficacy to 'prevent' a variety of pathological conditions, especially lifestyle-related diseases including cancer and 'metabolic syndrome'. If we rememeber that Chinese tea was actually a 'drug' in ancient East Asia which has now become a subject of serious study for the chemoprevention of cancer (6), this viewpoint will be well justified. I hope imaginative epidemiological studies in the East will track down interesting candidates for further analytical research among Kampo agents.

\section{Evaluation of Kampo Formulae on Disease Entities in the Modern Western Framework: Standard and Novel Clinical Trials}

\section{Standard Double-blind Randomized Control Trials (RCTs)}

The issues raised above are long-term and basic issues. However, Kampo medicine is now facing a more urgent and short-term challenge today. Society and governmental regulators are pressing for evidence to be presented regarding its efficacy. Simply put, there is clear demand that Kampo medicine should be evaluated on the same grounds and by the same methodology as synthetic drugs against disease entities in the modern Western framework. Many Kampo practitioners and researchers have been reluctant to comply with such demands, for the reasons repeated many times, namely paradigm differences related to the Sho concept. However, in the last decade, especially in Japan, there have been several attempts to evaluate Kampo agents in order to meet such demands. I would like to point out here that such a standard clinical trial is only possible if the drugs evaluated are highly homogeneous, and thus for Kampo herbs such quality control has only been possible for products from Japan.

As far as I am aware, there have been six reliable reports of standard double-blind randomized control trials on Kampo formulae after 1990, though unfortunately only one of them is completely in English (7). The report was from my own laboratory, examining the effect of Choto-san on vascular dementia. The result simply showed that Choto-san was more effective than placebo in 'general improvement' and 'usefulness' against vascular dementia. Other studies reported in Japanese with abstracts in English available are: Daiokanzoto on constipation (8), Shoseiryuto on nasal allergy (9) and Shakuyakukanzoto on muscular cramp (10). In all of these studies, Kampo agents were superior to placebo in a wide 


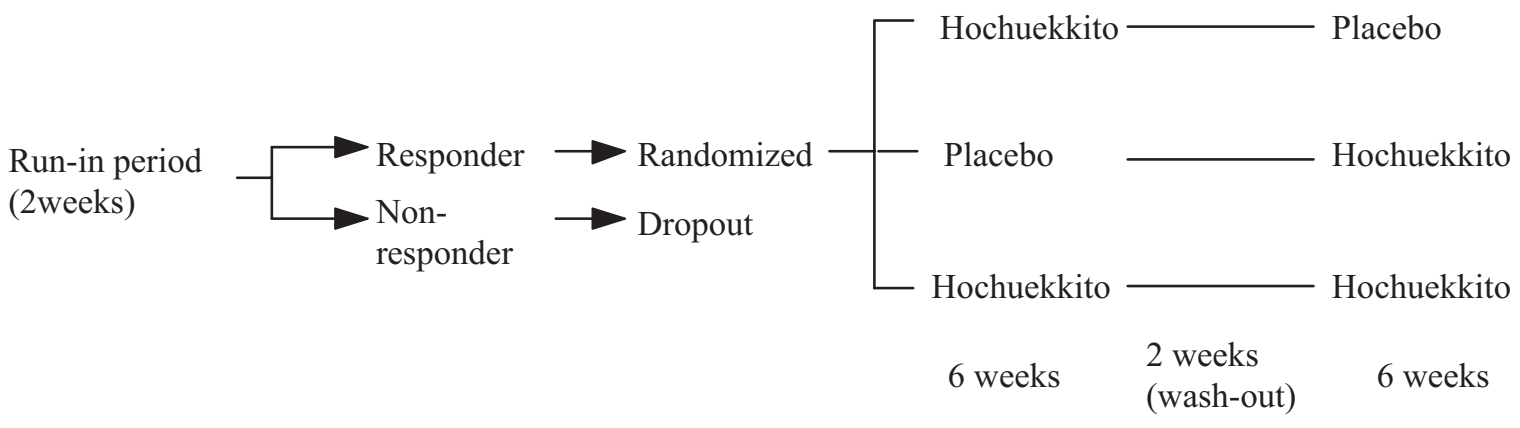

Figure 1. Conceptual protocol of a proposed clinical trial method for Kampo agents. After 2 weeks of a run-in period, responders to one Kampo agent (Hochuekkitoin, in this figure) are selected and enrolled into the actual trial. The trial consists of two periods with a 2 week interval. The responders are randomized into three groups: two groups taking the trial Kampo agent and one group taking placebo. In the second period, two groups taking either trial agent or placebo are changed to taking the other, while one group of patients taking the trial agent continues to take it.

variety of conditions. These results are of course not surprising at all, but it should be noted that there is no element of the authentic Kampo approach, namely that based on Sho, involved in these 'standard' trials. Kampo agents were just treated as 'drugs' in the modern Western framework. Many Kampo practitioners thought this approach was unfair.

\section{A Modified Double-blind RCT Based on Sho}

There was one report in 1998 by Harasawa and his collaborators, though again only in Japanese with no abstract in English, in which an RCT was attempted on a Kampo agent by taking Sho into account. Its study design was simple. The overall objective was to see if Rikkunshito is effective against dysmotility-like dyspepsia. In enrolling 235 subjects into the trial, the authors introduced inclusion and exclusion criteria where they included those who showed Sho indicative of Rikkunshito (which is Kyo-Sho) for the formula, but excluded those who showed Sho not indicative for Rikkunshito (such as muscular physique or ruddy face). As the formula has a peculiarly bitter 'Kampo' taste, the investigators mixed into the placebo a lower $(10 \%)$ dose of Rikkunshito. The results showed that the formula was significantly $(P=0.001)$ more 'effective' $(59.3 \%)$ than placebo (40.2\%) in improving the dysmotilitylike dyspepsia and also was more 'useful' (58.8 versus 39.3\%, $P=0.003)$. Interestingly, after stratified analysis, it was found that the formula was more effective $(P=0.001)$ when patients exhibited clearer Kyo-Sho (e.g. lowered muscular tonus in the abdomen) at the initial examination.

This type of trial ('pre-stratification according to Sho') is quite interesting and may look promising especially for researchers in the West. However, this type of trial design has again met with criticism from Kampo practitioners. This is basically because this design presupposes that Sho would not change during the trial, which is inconceivable in the Kampo paradigm. As explained in detail in the previous lecture, it is a rule and not an exception that Sho, being a concept of 'phenomenon', would change day by day, or even hour by hour. Such 'fluidity' in Sho makes a trial design such as that above difficult actually to carry out. The difficulty of incorporating Sho into rigid inclusion/exclusion criteria (such as age and sex) will be well understood if one could imagine that such phenomena or symptoms as 'fatigue' or 'malaise' are to be taken as inclusion/exclusion criteria in a formal RCT.

\section{New Trial Designs Being Investigated}

We have been trying to devise trial designs for Kampo, in which the main features of the Kampo approach are taken into considerations. I would like to outline one such design here (Fig. 1). In this scheme, first the Kampo agent being tested is tried for those whom the practitioners find to have its Sho (runin period). After 2 weeks, those who do not respond to the agent are excluded from the trial. In this way, only those who exhibit appropriate Sho are selected from the patients with the disease category for which the agent is tried. We call this process 'responder restriction' according to Sho. After this run-in period, patients are randomized into three groups: two groups with the real Kampo agent and one group with the placebo. After 6 weeks of the trial, the patients are assessed, and a 2 week wash-out period is introduced; subsequently, for the same three groups of patients, those who took placebo are changed to taking the real agent, while for one of the other two groups the real agent is changed for placebo. This is a sort of 'cross-over trial', where not only the effect of the Kampo agent is tested against placebo but in some of the participants the effect of the former is directly compared with the latter in individual patients. Thus, this scheme is regarded to be introducing the element of an ' $\mathrm{N}$ of one' trial method as proposed by Guyatt et al. (11). We think such a scheme would make sense in the Kampo setting, where response varies widely among individual patients and thus the effect of one kind of agent should be assessed against placebo in one individual patient.

\section{References}

1. Kobayashi H, Takahashi K, Mizuno N, Katsuna H, Ishii M. An alternative approach to atopic dermatitis: part I-case-series presentation. $e C A M$ 2004;1:49-62.

2. Kiyohara H, Matsumoto T, Yamada Y. Combination effects of herbs in a multi-herbal formula: expression of Juzen-taiho-to's immuno-modulatory activity on the intestinal immune system. eCAM 2004;1:83-91.

3. Terasawa K. Evidence-based reconstruction of Kampo medicine: part I-is Kampo CAM?. eCAM 2004;1:11-6. 
4. Terasawa K. Evidence-based reconstruction of Kampo medicine: part IIthe concept of Sho. eCAM 2004;2:118-23.

5. Tada T. Toward the philosophy of CAM: super-system and epimedical sciences. eCAM 2004;1:5-8.

6. Sasazuki S, Inoue M, Hanaoka T, Yamamoto S, Sobue T, Tsugano S. Green tea consumption and subsequent risk of gastric cancer by subsite: the JPHC study. Cancer Causes Control 2004;15:483-91.

7. Terasawa K, Shimada Y, Kita S et al. Choto-san in the treatment of vascular dementia: a double-blind, placebo-controlled study. Phytomedicine 1997;4:15-22.

8. Miyoshi A, Masamune $\mathrm{O}$, Fukutomi $\mathrm{H}$ et al. The clinical effect of TSUMURA Daio-Kanzo-to extract granules for ethical use (TJ-84) on constipation using double blind test. Shokakika (Gastroenterology) 1994; 18:299-311.

9. Baba $\mathrm{S}$, Takasaka $\mathrm{T}$, Inamura $\mathrm{N}$ et al. Double-blind clinical trial of Sho-seiryu-to (TJ-19) for perennial nasal allergy. Jibi-Rincho (Otolaryngology Clinics) 1995;88:389-405.

10. Kumada T, Kumada H, Yoshiba M, Nakano S, Suzuki H, Tango T. Effects of Shakuyaku-kanzo-to (Tsumura TJ-68) on muscle cramps accompanying cirrhosis in a placebo-controlled double-blind parallel study. Rincho-Yakuri (Clinical Pharmacology) 1999;15: 499-523.

11. Guyatt GH, Heyting A, Jaeschke R. N of 1 randomized trials for investigating new drugs. Controlled Clin Trials 1990;11:88-100. 


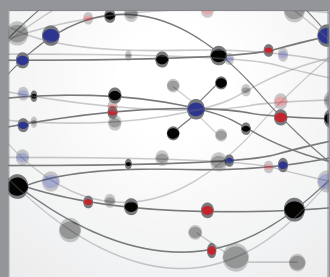

The Scientific World Journal
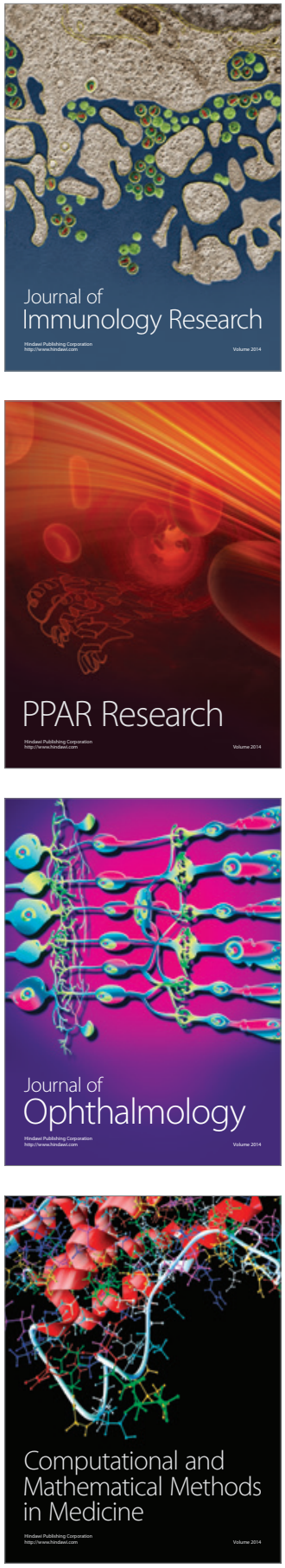

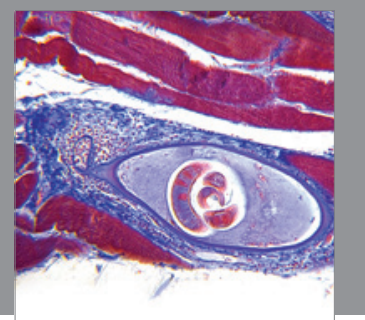

Gastroenterology

Research and Practice
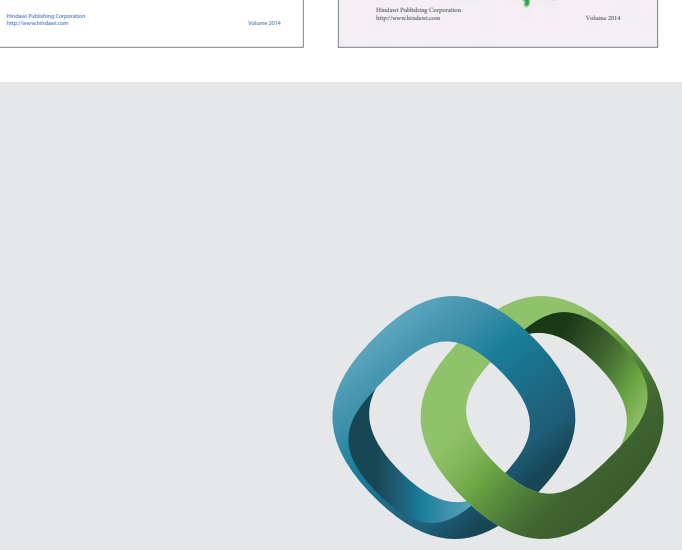

\section{Hindawi}

Submit your manuscripts at

http://www.hindawi.com
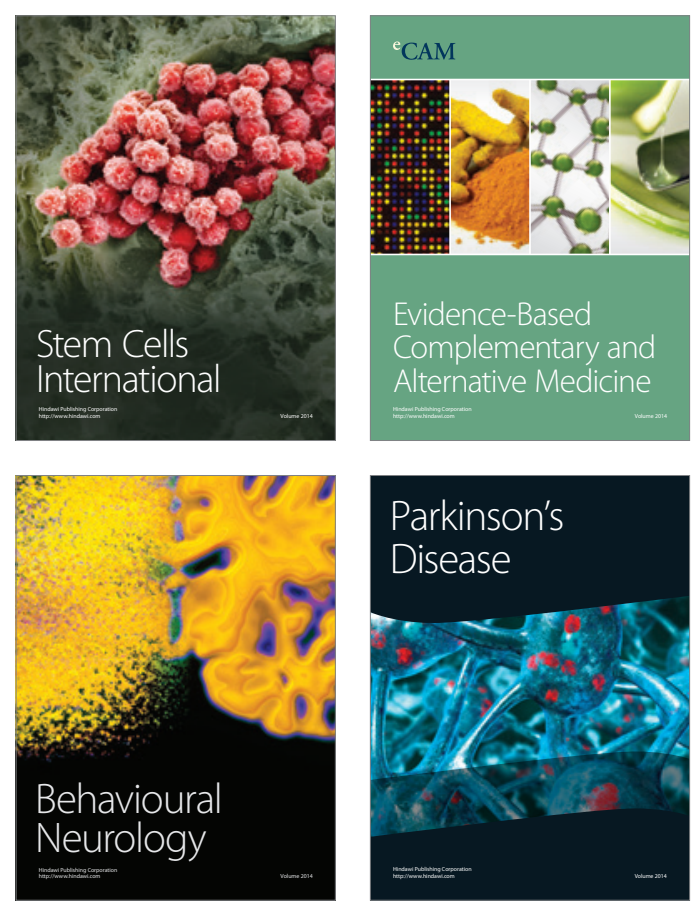

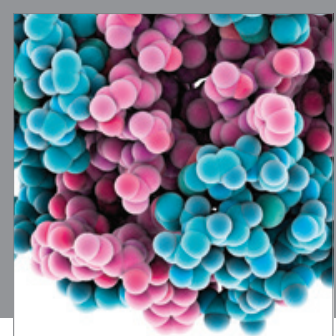

Journal of
Diabetes Research

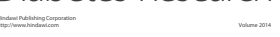

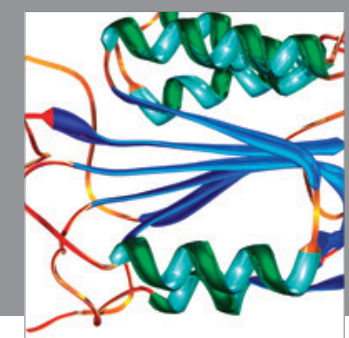

Disease Markers
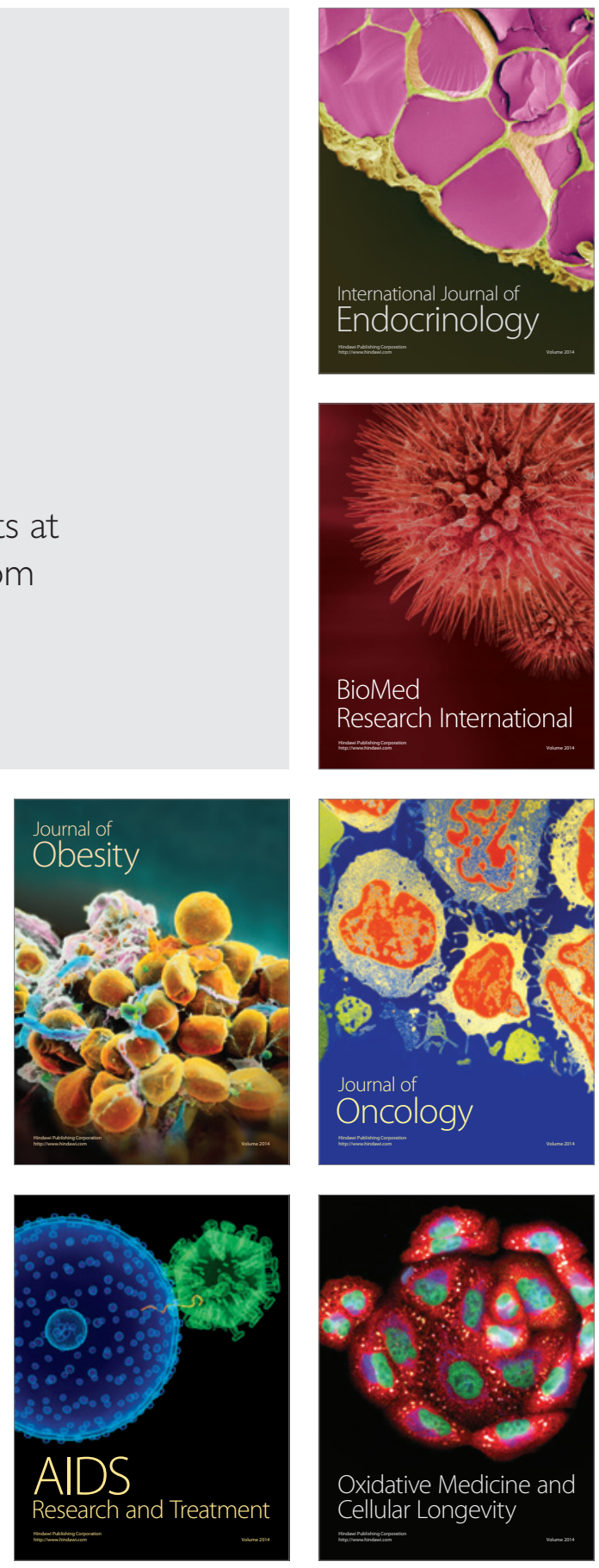